\title{
Hypothermia plus erythropoietin for neonatal neuroprotection? Commentary on Fan et al. and Fang et al.
}

\author{
Sandra E. Juul ${ }^{1}$
}

N eonatal neuroprotection for hypoxic-ischemic brain injury remains elusive. Preclinical studies of mild hypothermia in multiple animal models in several species (primarily, rodents, piglets, and sheep) showed significant benefit, and this therapy was therefore brought to clinical trials. Some of the preclinical trials showed little or no benefit, and these experiments allowed investigators to better define parameters in which hypothermia was effective. Important factors included timing and duration of therapy, depth of cooling, and use of anesthetics or morphine to prevent shivering. Multiple randomized controlled clinical trials of therapeutic hypothermia have now been completed, and meta-analyses of these trials definitively show benefit for infants with moderate to severe hypoxic-ischemic encephalopathy, with the number needed to treat between 7 and $9(1,2)$. In general, the benefits of hypothermia were greater in animal experiments than in human trials. This discrepancy is probably because preclinical experiments are carried out in otherwise healthy animals under controlled settings with the type, degree, and timing of injury all known. Used clinically, hypothermia improves both survival and the neurologic outcomes of those who survive, but the effect is only modest. Fifteen percent of cooled neonates with moderate to severe neonatal encephalopathy due to presumed hypoxia-ischemia still die, with $25 \%$ of qualifying infants suffering severe long-term neurodevelopmental impairment. Hence, the search continues for therapies that will further improve outcomes.

Erythropoietin (Epo) has great potential to be such an agent. In published preclinical studies, Epo has neuroprotective and neuroregenerative effects in the brain, with improvement rates after neonatal brain injury ranging from 34 to $79 \%$ (3). Mechanisms of Epo neuroprotection include receptor-mediated, cell-specific effects that occur both early and late in the healing process, and nonspecific effects that also modulate the response to injury. Epo has anti-inflammatory (4-6), antiexcitotoxic (7), antioxidant (8), and antiapoptotic (9) effects on neurons and oligodendrocytes (10). It also promotes neurogenesis $(11,12)$ and angiogenesis (13), which are essential for injury repair and normal neurodevelopment. Epo effects are dose dependent, and multiple doses are more effective than single doses $(9,14,15)$. The studies by Fang et al. (16) and Fan et al. (17) published in this issue both question whether Epo plus hypothermia might be more protective than either Epo or hypothermia alone. Surprisingly, the study by Fang et al. showed no benefit of $8 \mathrm{~h}$ of hypothermia; Epo treatment improved the histopathological outcome in males only, and combined therapy showed no benefit (or harm). In contrast, the study by Fan et al. showed neuroprotection after $3 \mathrm{~h}$ of hypothermia (greater effect in females than in males), improvement in sensorimotor function (but not amelioration of histopathological damage) with Epo alone, and combined therapy showed only modest benefit in sensorimotor function (observed in males only). How can we resolve these differences, and why are these studies discordant with previously published work?

The species and strain of animals used to model injury, mechanism of injury (stroke vs. hypoxia-ischemia), experimental design, and statistical issues can all affect outcomes. Cell-specific and regional vulnerability to brain injury changes with developmental stage, and these differ by species and strain; rats and mice have slightly different rates of brain development, and even within mice, different strains respond quite differently to hypoxia. Other less obvious factors might confound results. For example, unplanned maternal or neonatal stress (did the vivarium place barking dogs next to the rat room?) or unintentional changes in environmental factors (room temperature, noise, humidity, etc.) can affect outcomes.

So what factors might have affected the outcomes in the studies by Fang et al. and Fan et al.? The study by the former group was done in a laboratory that has previously shown robust Epo neuroprotection. Important differences between their current and previous studies include mechanism of injury (middle cerebral artery occlusion-no hypoxia vs. unilateral hypoxicischemic encephalopathy) and developmental stage, with the early studies using animals at postnatal day $(\mathrm{P}) 10$ rather than at P7 $(14,15)$. There are also several potentially important differences between the studies by Fang et al. and Fan et al. Although both studies used the Vannucci model of unilateral brain injury in P7 rats, the severity of injury differed (120 vs. $90 \mathrm{~min}$ of hypoxia) as did duration and degree of hypothermia ( 8 vs. $3 \mathrm{~h} ; 30.8^{\circ} \mathrm{C}$ cranial temperature vs. $32.5^{\circ} \mathrm{C}$ rectal temperature), the temperature of control animals $\left(33.8^{\circ} \mathrm{C}\right.$ cranial temperature vs. $36.5-37^{\circ} \mathrm{C}$ rectal temperature), Epo preparation (R\&D Systems vs. EPEX), Epo dose (1,000 vs. 5,000 U/kg), and 


\section{Commentary $\mid$ Commentary}

dosing intervals. Both studies mentioned power calculations, but it is not clear whether the extreme variability observed in the untreated brain-injured group was taken into account in these calculations. It appears that despite the prolonged period of hypoxia $(120 \mathrm{~min})$, some animals in the study by Fang et al. remained uninjured whereas others were severely affected. The uninjured animals increase within-group variability and ideally would be identified in real time and excluded from all treatment groups. It is possible that in such small treatment groups, these uninjured animals were randomly unevenly distributed, thus affecting the results. It is striking that neither therapy showed clinically significant neuroprotection in the Fang et al. study, suggesting there is something important to be learned about resistance to therapy.

Rodents are the most commonly used animals to model neonatal brain injury, so it is worthwhile considering some of the difficulties in translating information learned from rodents to humans. The rodent brain is lissencephalic, with a much smaller proportion of white matter than is present in humans. Foci of neurogenesis and timing of myelination are different. These factors may be important when studying the effect of an early insult on later brain development. The rate of maturation in a rat or mouse is accelerated relative to that in humans, with each day of rat development corresponding to more than a week of human development. However, the time course of response to injury appears to be similar in both species. Thus, as brain injury unfolds over hours to days, the developmental context changes differentially in rodents as compared with humans (as injury evolves from P7 to P10 in a rat, this time frame would roughly span $32 \mathrm{wk}$ to term in a human infant). Because the cellular and regional vulnerabilities of the brain vary by developmental stage, the effect of brain injury and its repair may be quite different in rodents than in humans. We do not know how these different time frames affect dosing duration and dosing interval, or how response to therapeutics interacts with the evolution of injury. For example, in humans and larger animal models, it is known that $72 \mathrm{~h}$ of hypothermia is more beneficial than 12 or $24 \mathrm{~h}$. How does this translate to rat pups? Are 3 or 8 h sufficient? Or are 24 or $72 \mathrm{~h}$ required for neuroprotection? Therapy should optimally target the timing of response to injury, the pattern of cell death, and inflammatory response, followed by repair. Differences in drug metabolism may also be important when translating preclinical trials to humans. We have seen that in extremely low birth weight infants, $500 \mathrm{U} / \mathrm{kg}$ Epo IV results in peak circulating concentrations similar to those achieved in rat pups given $5,000 \mathrm{U} / \mathrm{kg}$ i.p., but area under the curve is most similar when $1,000 \mathrm{U} / \mathrm{kg}$ in a premature infant is compared with 5,000 U/kg in a rat $(18,19)$.

In human studies, we do not consider a therapy proven until there are many hundreds (or even thousands) of subjects who have shown benefit. Yet in animal studies, we expect to show meaningful differences comparing small groups. This may not be a reasonable expectation. At this point, more studies are needed, ideally, in multiple large and small animal models to establish whether hypothermia and Epo will prove to be of additional benefit relative to hypothermia alone.

\section{REFERENCES}

1. Edwards AD, Brocklehurst P, Gunn AJ, et al. Neurological outcomes at 18 months of age after moderate hypothermia for perinatal hypoxic ischaemic encephalopathy: synthesis and meta-analysis of trial data. BMJ 2010;340:c363.

2. Tagin MA, Woolcott CG, Vincer MJ, Whyte RK, Stinson DA. Hypothermia for neonatal hypoxic ischemic encephalopathy: an updated systematic review and meta-analysis. Arch Pediatr Adolesc Med 2012;166:558-66.

3. van der Kooij MA, Groenendaal F, Kavelaars A, Heijnen CJ, van Bel F. Neuroprotective properties and mechanisms of erythropoietin in in vitro and in vivo experimental models for hypoxia/ischemia. Brain Res Rev 2008;59:22-33.

4. Sun Y, Calvert JW, Zhang JH. Neonatal hypoxia/ischemia is associated with decreased inflammatory mediators after erythropoietin administration. Stroke 2005;36:1672-8.

5. Juul SE, Beyer RP, Bammler TK, McPherson RJ, Wilkerson J, Farin FM. Microarray analysis of high-dose recombinant erythropoietin treatment of unilateral brain injury in neonatal mouse hippocampus. Pediatr Res 2009;65:485-92.

6. Rees S, Hale N, De Matteo R, et al. Erythropoietin is neuroprotective in a preterm ovine model of endotoxin-induced brain injury. J Neuropathol Exp Neurol 2010;69:306-19.

7. Zacharias R, Schmidt M, Kny J, et al. Dose-dependent effects of erythropoietin in propofol anesthetized neonatal rats. Brain Res 2010;1343:14-9.

8. Kumral A, Gonenc S, Acikgoz O, et al. Erythropoietin increases glutathione peroxidase enzyme activity and decreases lipid peroxidation levels in hypoxic-ischemic brain injury in neonatal rats. Biol Neonate 2005;87:15-8.

9. Kellert BA, McPherson RJ, Juul SE. A comparison of high-dose recombinant erythropoietin treatment regimens in brain-injured neonatal rats. Pediatr Res 2007;61:451-5.

10. Iwai M, Stetler RA, Xing J, et al. Enhanced oligodendrogenesis and recovery of neurological function by erythropoietin after neonatal hypoxic/ ischemic brain injury. Stroke 2010;41:1032-7.

11. Wang L, Zhang Z, Wang Y, Zhang R, Chopp M. Treatment of stroke with erythropoietin enhances neurogenesis and angiogenesis and improves neurological function in rats. Stroke 2004;35:1732-7.

12. Xiong Y, Mahmood A, Meng Y, et al. Delayed administration of erythropoietin reducing hippocampal cell loss, enhancing angiogenesis and neurogenesis, and improving functional outcome following traumatic brain injury in rats: comparison of treatment with single and triple dose. J Neurosurg 2010;113:598-608.

13. Wang L, Chopp M, Gregg SR, et al. Neural progenitor cells treated with EPO induce angiogenesis through the production of VEGF. J Cereb Blood Flow Metab 2008;28:1361-8.

14. Gonzalez FF, McQuillen P, Mu D, et al. Erythropoietin enhances longterm neuroprotection and neurogenesis in neonatal stroke. Dev Neurosci 2007;29:321-30.

15. Gonzalez FF, Abel R, Almli CR, Mu D, Wendland M, Ferriero DM. Erythropoietin sustains cognitive function and brain volume after neonatal stroke. Dev Neurosci 2009;31:403-11.

16. Fang AY, Gonzalez FF, Sheldon RA, Ferriero DM. Effects of combination therapy using hypothermia and erythropoietin in a rat model of neonatal hypoxia-ischemia. Pediatr Res 2013;73:12-7.

17. Fan X, van Bel F, van der Kooij MA, J. Heijnen CJ, Groenendaal F. Hypothermia and erythropoietin for neuroprotection after neonatal brain damage. Pediatr Res 2013;73:18-23

18. Juul SE, McPherson RJ, Bauer LA, Ledbetter KJ, Gleason CA, Mayock DE. A phase I/II trial of high-dose erythropoietin in extremely low birth weight infants: pharmacokinetics and safety. Pediatrics 2008;122:383-91.

19. Statler PA, McPherson RJ, Bauer LA, Kellert BA, Juul SE. Pharmacokinetics of high-dose recombinant erythropoietin in plasma and brain of neonatal rats. Pediatr Res 2007;61:671-5. 